www.nature.com/pj

\title{
Layer-by-layer fabrication of well-packed gold nanoparticle assemblies guided by a $\beta$-sheet peptide network
}

\author{
Nobuyuki Higashi, Toshikazu Takagi and Tomoyuki Koga
}

In this study, we describe the first demonstration of well-ordered multilayer thin films of gold nanoparticles through a layer-bylayer (LbL) technique assisted by a polypeptide template. Anionic poly(L-glutamic acid) (PLGA)-modified gold nanoparticles were prepared as building units for LbL self-assembly. Two types of synthetic polymers, poly(L-lysine) (PLL) and poly(allylamine), were used as polycations that carry the same amino groups at the side chain, although the former is a polypeptide and the latter is a vinyl polymer. The deposition process and the resultant multilayer films were characterized by means of absorption, circular dichroism, Fourier transform infrared and atomic force microscopy measurements. Experimental results revealed that LbL multilayer properties, such as thickness, conformation of the peptide component, homogeneity and periodicity of nanoparticle layers, were significantly affected by the kind of polycations present. In particular, the combination of anionic PLGA-coated gold nanoparticles and cationic PLL induced $\beta$-sheet formation, which enabled the fabrication of a homogeneously well-packed gold nanoparticle multilayer without aggregation.

Polymer Journal (2010) 42, 95-99; doi:10.1038/pj.2009.311

Keywords: Au nanoparticle; layer-by-layer deposition; multilayer; polypeptide; $\beta$-sheet; structural color

\section{INTRODUCTION}

Particles in the nanometer size range attract increasing attention with the growth of interest in nanotechnological disciplines. Nanoparticles show fascinating optical and electronic properties as a consequence of their dimensions. The dimensions of these particles make them ideal candidates for the fabrication of functional nanostructures. To tailor the new generation of nanomaterials, it is essential to organize nanoparticles into controlled architectures. The construction of two-dimensional particle arrays is well studied. ${ }^{1}$ The groundwork has led to methods for the preparation of more complex three-dimensional systems, which may eventually lead to highly functionalized assemblies and composites. In this study, we describe the fabrication of well-controlled multilayers of gold nanoparticles using specific conformations of peptides as a template. In particular, $\beta$-sheet peptides are promising candidates for such a template or matrix because they can often be seen in natural fibers as a structural element. In fact, the Langmuir monolayers of $\beta$-sheet peptides were reported to work as templates and nucleate the formation of CdS nanocrystallites at the air-water interface. ${ }^{2}$ Recently, we demonstrated that an amphiphilic artificial peptide can self-assemble into $\beta$-sheet nanofiber architectures, ${ }^{3-5}$ the surfaces of which have potential as a novel nanotemplate. ${ }^{6}$ Stupp's group ${ }^{7}$ and Mihara's group ${ }^{8}$ used $\beta$-sheet peptide nanofibers with surface-binding sites as nano- templates to accomplish the one-dimensional organization of functional materials such as gold nanoparticles and proteins. Previously, we successfully prepared a poly( $\gamma$-benzyl-L-glutamate)-coated gold nanoparticle that was stably dispersed in organic solvents without any aggregation. ${ }^{9}$

In this paper, we fabricated multilayered films of gold nanoparticles coated with anionic poly(L-glutamic acid), and polycations, using the layer-by-layer (LbL) technique. ${ }^{10,11}$ Among inorganic (or metallic) and polymeric nanoparticles, gold nanoparticles have an easily modified surface and are detectable spectroscopically because of their surface plasmon resonance. In addition, the surface plasmon resonance of gold nanoparticles gives them great potential to be used for various applications such as catalysts, nanoelectronic components, nonlinear optical devices and solid-state chemical and biological sensors, but these applications also require the immobilization of gold nanoparticles from colloidal solutions onto solid substrates without aggregation. ${ }^{12-14}$ Aggregation would cause the nanoparticles to lose their own unique size-dependent physical and chemical properties. As polycations, poly(L-lysine) (PLL) and poly(allylamine) (PAA) are used, which are a polypeptide and a vinyl polymer, respectively, but they have the same pendant amino group. The LbL assembly takes advantage of the same attractive forces that form complexes but in a controlled manner that produces thin, conformal 
films that can coat a variety of surfaces and thus are a powerful method for fabricating multilayer gold nanoparticle films. ${ }^{15-27}$ In spite of these many reports, to the best of our knowledge, well-controlled gold nanoparticle assemblies guided with a polypeptide network have not yet been reported. The aim of this article is to construct homogeneously well-ordered gold nanoparticle multilayers assisted with a $\beta$-sheet peptide template.

\section{EXPERIMENTAL PROCEDURE}

\section{Materials}

$\mathrm{HAuCl}_{4}, \mathrm{PLL}(\mathrm{Mw}=50000)$, PAA $(\mathrm{Mw}=70000)$ and other chemicals were used as received.

Disulfide bond-attached $\operatorname{poly}(\gamma$-benzyl-L-glutamate $)$ was first synthesized by the NCA-polymerization method, according to the manner reported previously, ${ }^{9}$ and then the removal of the benzyl group in the side chain by $5 \% \mathrm{HBr} / \mathrm{CH}_{3} \mathrm{COOH}$ resulted in the objective disulfide bond-attached poly(L-glutamic acid) (PLGA-SS). The degree of polymerization ( $n$ ) was 18 , as determined by ${ }^{1} \mathrm{H}-\mathrm{NMR}$ spectroscopy. Monodispersed PLGA-SS-coated gold nanoparticles (1) were prepared in the presence of PLGA-SS by sodium borohydride $\left(\mathrm{NaBH}_{4}\right)$. Particle diameter was evaluated to be $20 \pm 4 \mathrm{~nm}$ by transmission electron microscopy (H-8100, Hitachi, Tokyo, Japan).

\section{LbL assembly}

The preparation procedures of LbL assemblies are outlined in Figure 1. A quartz plate was sonicated in a mixture of concentrated $\mathrm{H}_{2} \mathrm{SO}_{4}$ and $30 \% \mathrm{H}_{2} \mathrm{O}_{2}$ aqueous ( $7: 3 \mathrm{in}$ vol.) for $30 \mathrm{~min}$; thereafter, the plate was washed with pure water and dried. The plate was first immersed into a $5 \mathrm{mM} \mathrm{CHCl}_{3}$ solution of 3-aminopropyltriethoxysilane overnight at room temperature, washed with pure $\mathrm{CHCl}_{3}$ and dried, after which it was incubated in a phosphate-buffered solution at $\mathrm{pH} 8.0$ for $1 \mathrm{~h}$, so that the immobilized amino groups were completely protonated and the surface carried multiple positive charges (step 1). The plate was immersed in the phosphate-buffered solution of

\section{a PLGA-modified gold nanoparticle (1)}

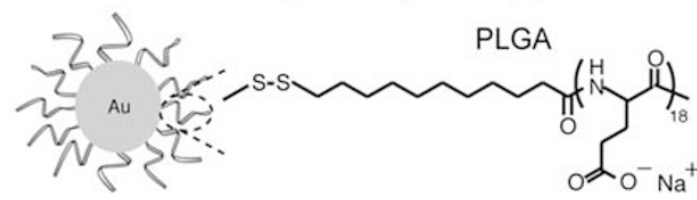<smiles>CCC(=O)C(CCCC[NH3+])NC(C)(C)C</smiles>

PLL

b

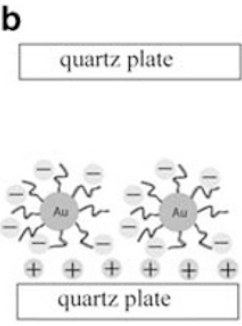

$$
\underset{\text { step } 1}{\stackrel{\text { APTES }}{\longrightarrow}}
$$

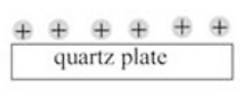

$\operatorname{step} 2$

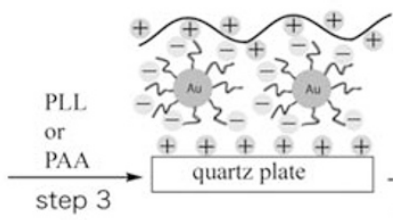

PLL

or PAA step 2 step 3
Figure 1 Chemical structures of the component molecules used in this study (a) and preparation procedures of alternate assemblies (b). $1\left(0.1 \mathrm{mg} \mathrm{ml}^{-1}\right)$ at $\mathrm{pH} 8.0$ (step 2), followed by washing with the same buffer. Next, the 1-immobilized plate was immersed in the phosphate-buffered solution of polycation $\left(0.1 \mathrm{mg} \mathrm{ml}^{-1}\right)$ at $\mathrm{pH} 8.0$ (step 3), followed by washing with the same buffer. By repeating steps 2 and 3, multilayered films of 1 and PLL or PAA were obtained.

\section{Characterization}

Characterizations of deposition processes and the resultant multilayer films were undertaken by means of absorption (UV2450, Shimadzu, Kyoto, Japan), circular dichroism (CD) (J-720, JASCO, Tokyo, Japan) and Fourier transform infrared (Nexus 470, Thermo Nicolet, Madison, WI, USA) spectroscopies and atomic force microscopy (Nanoscope IIIa, Digital Instruments, Santa Barbara, CA, USA).

\section{RESULTS AND DISCUSSION}

The PLGA-SS-coated gold nanoparticles (1) were dissolved in aqueous solutions with a wide range of $\mathrm{pHs}(4-11)$ and formed stable colloidal solutions. The secondary structure of these colloidal solutions was evaluated by CD spectroscopy. At $\mathrm{pH} 4.0$, the CD spectrum showed the typical $\alpha$-helical conformation of PLGA with two negative peaks at 210 and $220 \mathrm{~nm}$. With elevated $\mathrm{pH}$, the spectra showed a drastic change from helices to random coils at around $\mathrm{pH}$. These $\mathrm{pH}-$ dependent spectral variations are very similar to those for gold-free PLGA. ${ }^{28}$

Figure 2 shows ultraviolet-visible spectra of 1/PLL films as a function of the number of dipping cycles. The quartz plate was immersed into each solution for $60 \mathrm{~min}$, after which adsorption was completely at equilibrium. The surface plasmon resonance bands for gold nanoparticles in films are similar in shape to those for isolated nanoparticles in the colloidal solution. The absorption maxima of these bands $\left(\lambda_{\max }\right)$ were fixed at a wavelength of $542 \mathrm{~nm}$ and did not

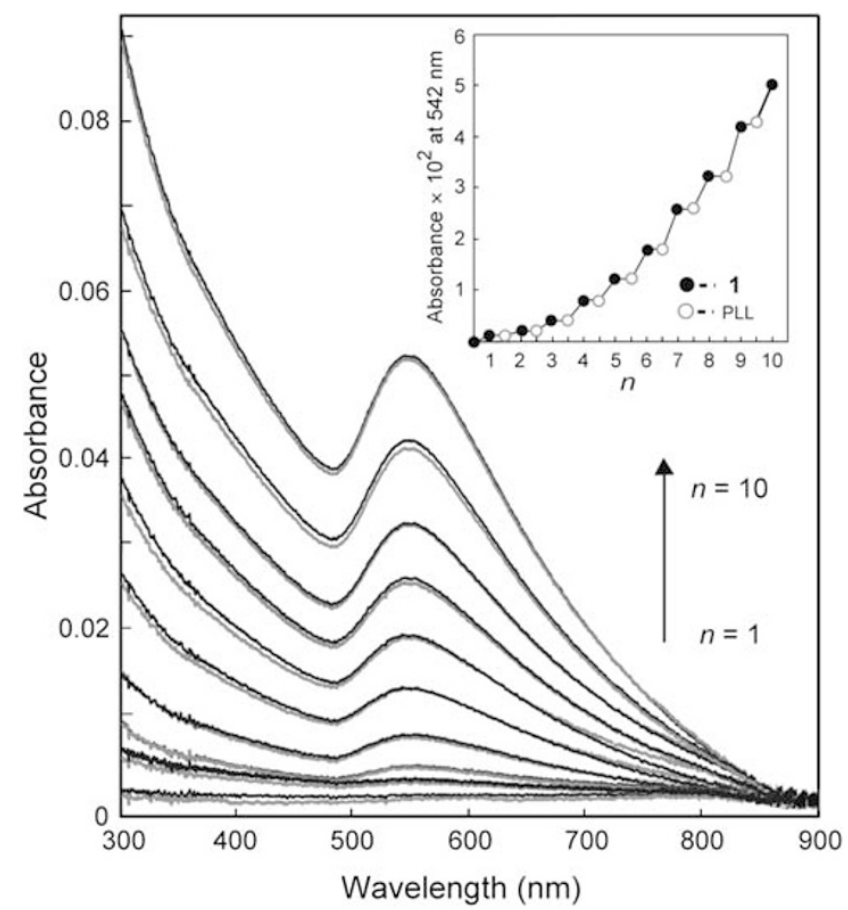

Figure 2 Surface plasmon resonance absorption spectra of a 1/poly(L-lysine) (PLL) multilayer film (black line) as a function of the number of dipping cycles. The spectrum of a 1-colloidal solution is also shown as a gray line for comparison. The inset shows the absorbance change at $542 \mathrm{~nm}$ after alternate immersion into the 1-colloidal solution (black circle) and the aqueous PLL solution (white circle). The abscissa ' $n$ ' indicates the number of a set of 1/PLL assemblies. 
change with the number of dipping cycles. Previous reports ${ }^{21-27}$ have pointed out that in multilayer gold nanoparticles, strong interactions between neighboring dipolar particles can readily take place, which cause a red shift in $\lambda_{\max }$ with increasing number of dipping cycles and/or generate a new absorption band at a longer wavelength. In our case, however, no such features appear in the spectra, as shown in Figure 2, suggesting that the gold nanoparticles in films are spatially separated from each other, probably because of the long PLGA chains attached to the gold nanoparticles, and that the interparticle interactions are relatively weak. The slight red shift observed from $534 \mathrm{~nm}$ for the solution to $542 \mathrm{~nm}$ for the films might be ascribed to the change in the dielectric environment and to the weak inter-particle interactions in films. The inset in Figure 2 shows absorbance changes at $\lambda_{\max }=542 \mathrm{~nm}$ after immersion alternatively in the $\mathbf{1}$ colloidal solution and aqueous PLL solution at $\mathrm{pH}$ 8.0. Stepwise increases in absorbance after immersion in the $\mathbf{1}$ colloidal solution clearly indicate the adsorption of $\mathbf{1}$ on the substrate, except for an initial dipping process. By contrast, immersion in the aqueous solutions of PLL shows no significant change in absorbance at $542 \mathrm{~nm}$, although absorbance at $200 \mathrm{~nm}$ because of peptide bonds increased smoothly. The 1/PAA assembly also showed stepwise increases in absorbance at $542 \mathrm{~nm}$ but showed no change in absorbance at $200 \mathrm{~nm}$ because PAA has no peptide bonds. These results indicate that the alternative $\mathrm{LbL}$ deposition successfully proceeds for both combinations of $\mathbf{1}$ and PLL or PAA.

Figure 3 shows the CD spectra of deposited 1/PLL and 1/PAA films. In the case of $1 /$ PLL (Figure $3 \mathrm{a}$ ), the spectra give a $\beta$-sheet pattern with a single negative maximum at $218 \mathrm{~nm}$, and the peak intensity increases with increasing layer number. When an attenuated total reflectance-Fourier transform infrared measurement was performed for the 10-layer 1/PLL film in the amide I and II regions, characteristic absorptions were observed at 1615 and $1532 \mathrm{~cm}^{-1}$, respectively (Figure 4a), indicating the formation of $\beta$-sheet conformation. ${ }^{29}$ A peak deconvolution of amide I and II bands resulted in the following content of secondary structures: $75 \% \beta$-sheet, $20 \% \alpha$ helix and 5\% random coil. From these spectroscopic data, it can be concluded that inter-polypeptide interactions have taken place between the PLGA of 1 and PLL during the LbL deposition process and as a consequence led to $\beta$-sheet conformation through multiple hydrogen-bonding interactions, as each polypeptide segment in the solution originally has the random coil conformation under these conditions. In contrast, CD spectra of $1 / \mathrm{PAA}$ (Figure $3 \mathrm{~b}$ ) give a typical $\alpha$-helix pattern with two negative maxima at 210 and $222 \mathrm{~nm}$, although the peak intensity systematically increases with deposition. The attenuated total reflectance-Fourier transform infrared spectrum of the 10-layer film also supported the $\alpha$-helical conformation with a content of $75 \%$ (Figure $4 \mathrm{~b}$ ). The results suggest that inter-polymer interactions of PLGA and PAA may not be as strong as those of PLGA and PLL. PAA is a vinyl polymer that does not form such specific secondary conformations as $\alpha$-helices or $\beta$-sheets. Thus, the negatively charged PLGA segments of $\mathbf{1}$ were partially neutralized with cationic PAA through electrostatic interaction, and their microenvironment changed into one likely to adopt the $\alpha$-helical conformation, which is more favorable for PLGA by nature. To obtain information about the surface of these deposited films, atomic force microscopy observation was performed, and the results are compared in Figure 5. Pictures show tapping-mode atomic force microscopy images of the outermost surfaces of 10-layer 1/PLL and 1/PAA. The last step of deposition was accomplished by dipping the substrate into the $\mathbf{1}$ colloidal solution. Therefore, the outermost surfaces must be occupied by 1-layer. As a matter of fact, in Figure 5a, homogeneous, densely dispersed nano-
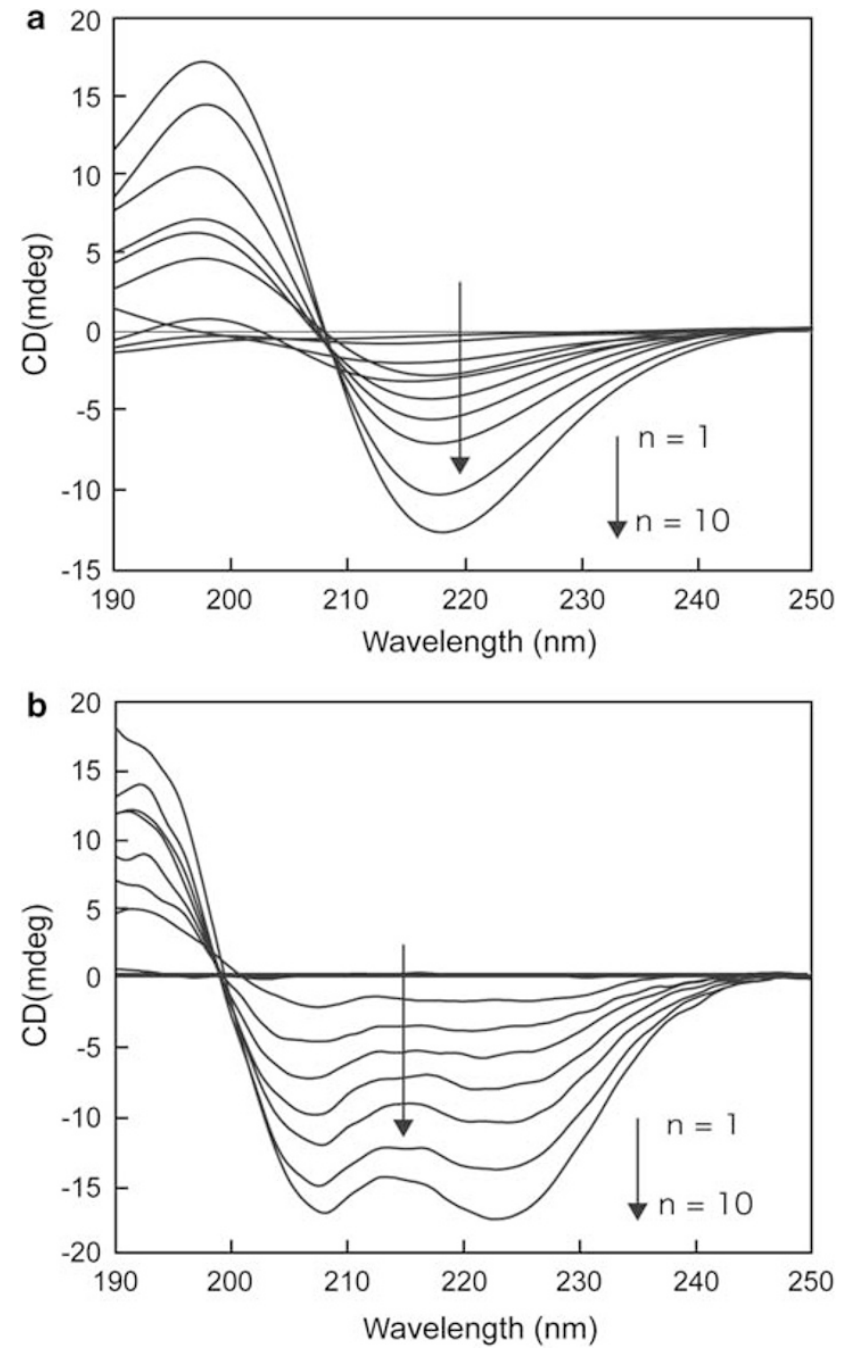

Figure 3 Circular dichroism spectral changes for the combinations of 1/poly(L-lysine) (a) and 1/poly(allylamine) (b) during layer-by-layer deposition.

particles can be clearly observed. In Figure 5b, by contrast, there are not only loosely packed nanoparticles but also somewhat huge domains as a consequence of the heterogeneous aggregation of gold particles. Subsequently, we tried to determine film thickness by comparing the height profile in atomic force microscopy for a deposited region with that for a bare region on the same substrate. The thickness of the 1/PLL film was estimated to be $210 \pm 40 \mathrm{~nm}$, which corresponds to the thickness of very dense, 10-layer gold nanoparticles, as their core diameter was evaluated to be $20 \pm 4 \mathrm{~nm}$ by transmission electron microscopy observation, as previously mentioned. The 1/PAA assembly provided a much thicker film of $690 \pm 50 \mathrm{~nm}$ in spite of having the same layer number as the 1/PLL assembly.

Finally, we examined the structural color formation of these deposited films, which is an effective characterization method to clarify the regularity and/or homogeneity of layered structures at the nanoscale. Silicon wafers were used as substrate in place of quartz plates for this purpose and treated at $1100{ }^{\circ} \mathrm{C}$ for $4 \mathrm{~h}$ to transform the surface to an oxide before use, so that the interference color was more easily detectable. ${ }^{30}$ The deposition procedure was the same as that on the quartz plate. The substrates show various interference colors 
a

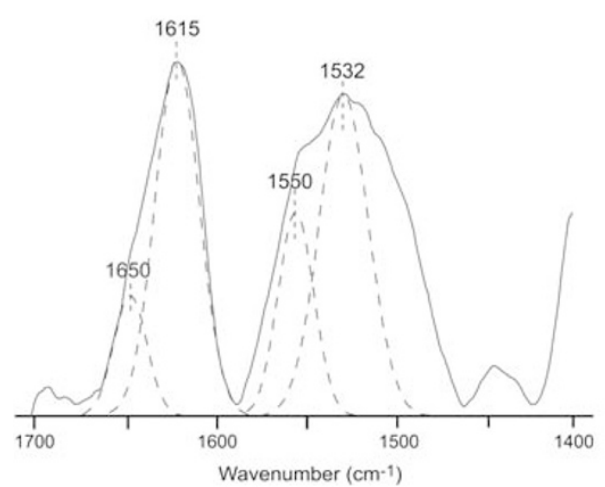

b

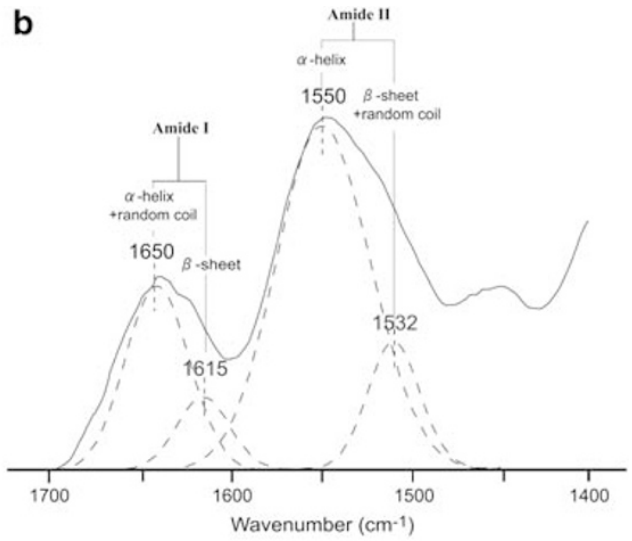

Figure 4 Attenuated total reflectance-Fourier transform infrared spectra for 10-layered 1/poly(L-lysine) (a) and 1/poly(allylamine) (b) films.
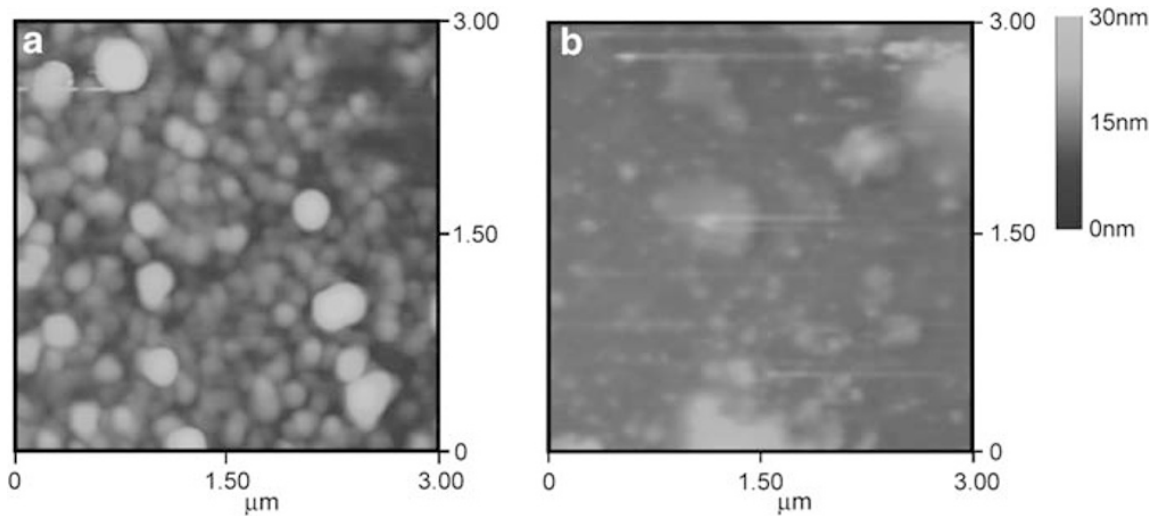

Figure 5 Tapping-mode atomic force microscopy images for the outermost surfaces of 10-layered 1/poly(L-lysine) (PLL) (a) and 1/poly(allylamine) (PAA) (b) films.

depending on the number of layers (Figure 6B). Particularly, the photograph of 1/PLL films clearly shows a layer number-dependent color change: bluish green at 5 layers, yellow green at 10 layers and red-purple at 15 layers. However, 1/PAA films do not show such a clear color change because of the layer number and instead show a lack of uniformity in color. This result also supports the assertion that the layered structure of $1 /$ PLL films is well controlled and has a favorable periodic regularity due to the $\beta$-sheet network, compared with that of 1/PAA films.

Figure $6 \mathrm{~A}$ shows plausible models for the cross-sectional view of deposited films on the basis of the results obtained above. The overall film thickness was considerably different between 1/PLL and 1/PAA, although the absorbance of the surface plasmon resonance band for 10-layer films was close to each other (data not shown), suggesting the difference in the thickness of polycation layers. As illustrated in the figure, the complexation of 1 with PAA through partial electrostatic interaction seems to bring about the $\alpha$-helical conformation of PLGA segments that is originally inherent and simultaneously leaves many unreacted cations on PAA molecules, which provide an expanded conformation. In contrast, the complexation of 1 with PLL leads to a very dense, well-packed gold nanoparticle multilayer, probably because electrostatic interactions work at first between PLGA and PLL and may be a driving force for making inter-molecular hydrogen bonds that induce $\beta$-sheet network formation. a layers

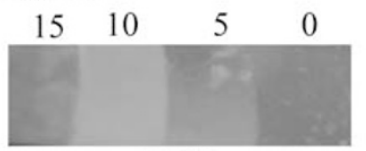

1/PLL

b

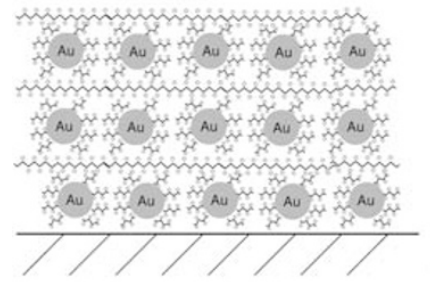

1/PLL layers
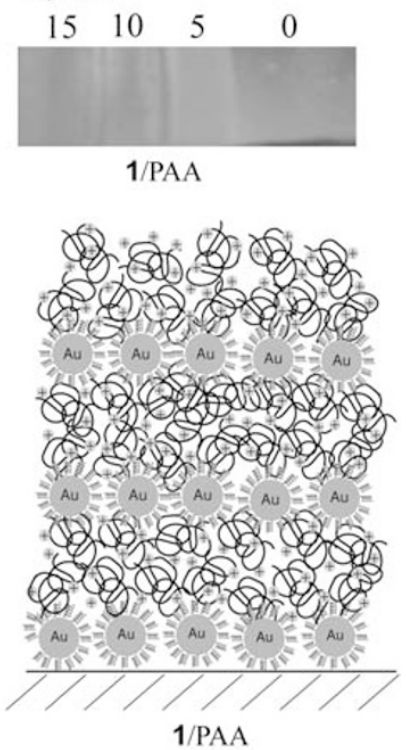

1/PAA
Figure 6 Photographs of 1/PLL and 1/PAA multilayer films on oxide silicon plates (a) and plausible models of 1/PLL and 1/PAA multilayer films (b). 


\section{CONCLUSION}

In this study, we described the first demonstration of the LbL selfassembly of multilayer thin films of gold nanoparticles assisted with a $\beta$-sheet peptide template. Multilayer films were successfully prepared by the combined use of anionic PLGA-coated gold nanoparticles and polycations and were characterized using various techniques. As a polycation, we used two types of synthetic polymers, PLL and PAA, which carry the same $\mathrm{NH}_{2}$ group as a side chain, although the former is a polypeptide and the latter is a vinyl polymer. Characterizations of the resultant multilayer films reveal that the dominant properties of films, such as film thickness, the secondary structure of the peptide component, as well as the homogeneity and periodicity of nanoparticle layers, are significantly affected by the kind of polycation used. In particular, when we used PLL as the polycation, the inter-molecular $\beta$-sheet structure can be spontaneously set up by an electrostatic interaction of PLGA segments with gold nanoparticles and PLL during the deposition process. This $\beta$-sheet network makes it possible to fabricate a homogeneously well-packed gold nanoparticle multilayer without aggregation.

\section{ACKNOWLEDGEMENTS}

This work was supported in part by the Projects 'Creating Research Center for Advanced Molecular Biochemistry' and 'Research Center for Interfacial Phenomena' from the Ministry of Education, Culture, Sports, Science and Technology (MEXT) Japan, and by a grant-in-aid for young scientific research (no. 21750130) from the MEXT.

1 Shipway, N. A., Katz, E. \& Willner, I. Nanoparticle array on surfaces for electronic, optical and sensing applications. Chem. Phys. Chem. 1, 18-52 (2000) and references cited therein

2 Bekele, H., Fendler, H. J. \& Kelly, W. J. Self-assembling peptidomimetic monolayer nucleates CdS nanocrystals. J. Am. Chem. Soc. 121, 7266-7267 (1999).

3 Koga, T., Matsuoka, M. \& Higashi, N. Structural control of self-assembled nanofibers by artificial $\beta$-sheet peptides composed of $d$ - or l-isomer. J. Am. Chem. Soc. 127, 17596-17597 (2005)

4 Koga, T., Higuchi, M., Kinoshita, T. \& Higashi, N. Controlled self-assembly of amphiphilic oligopeptides into shape-specific nanoarchitectures. Chem. Eur. J. 12, 1360-1367 (2006).

5 Koga, T., Kitamura, K. \& Higashi, N. Enzymatically Triggered Self-assembly of Poly(ethylene glycol)-attached Oligopeptides into Well-organized Nanofibers. Chem. Commun. 4897-4898 (2006).

6 Koga, T., Murakami, R. \& Higashi, N. Surface modification of peptide nanofiber by using antigen-antibody interaction. Trans. Mater. Res. Soc. Jpn. 32, 371-374 (2007).

7 Li, L. \& Stupp, J. S. Surface modification of peptide nanofiber by using antigenantibody interaction. Angew. Chem. Int. Ed. 44, 1833-1836 (2005).

8 Kodama, H., Matsumura, S., Yamashita, T. \& Mihara, H. Construction of a protein array on amyloid-like fibrils using co-assembly of designed peptides. Chem. Commun. 2876-2877 (2004)
9 Higashi, N., Kawahara, J. \& Niwa, M. Preparation of helical peptide monolayer-coated gold nanoparticles. J. Colloid Interface Sci. 288, 83-87 (2005).

10 Decher, G. \& Hong, J. Consecutively alternating adsorption of anionic and cationic polyelectrolytes on charged surface. Thin Solid Films 210, 831-835 (1992).

11 Decher, G. Fuzzy nanoassemblies: toward layered polymeric multicomposites. Science 277, 1232-1237 (1997).

12 Feldheim, L. D., Grabar, C. K., Natan, J. M. \& Mallouk, E. T. Electron transfer in selfassembled inorganic polyelectrolyte/metal nanoparticle heterostructures. J. Am. Chem. Soc. 118, 7640-7641 (1996).

13 Nath, N. \& Chilkoti, A. A colorimetric gold nanoparticle sensor to interrogate biomolecular interactions in real time on a surface. Anal. Chem. 74, 504-509 (2002)

$14 \mathrm{Li}, \mathrm{D} ., \mathrm{He}, \mathrm{Q}$., Cui, Y. \& Li, J. Fabrication of $\mathrm{pH}$-responsive nanocomposites of gold nanoparticles/Poly(4-vinylpyridine). Chem. Mater. 19, 412-417 (2007).

15 Mirkin, A. C., Letsinger, L. R., Mucic, C. R. \& Storhoff, J. J. A DNA-based method for rationally assembling nanoparticles into macroscopic materials. Nature 382, 607-609 (1996).

16 Zhou, H., Aoki, S., Honma, I., Hirasawa, M., Nagamura, T. \& Komiyama, H. Conformational change of protein cytochrome b-562 adsorbed on colloidal gold particles; absorption band shift. Chem. Commun. 605-606 (1997).

17 Sastry, M., Lala, N., Patil, V., Chavan, P. S. \& Chittiboyina, G. A. Optical absorption study of the biotin-avidin interaction on colloidal silver and gold particles. Langmuir 14, 4138-4142 (1998).

18 Liu, Y., Wang, Y. \& Claus, O. R. Layer-by-layer ionic self-assembly of au colloids into multilayer thin-films with bulk metal conductivity. Chem. Phys. Lett. 298, 315-319 (1998).

19 Schmitt, J., Machtle, P., Eck, D., Mohwald, H. \& Helm, A. C. Preparation and optical properties of colloidal gold monolayers. Langmuir 5, 3256-3266 (1999).

20 Baum, T., Brust, D., Bethell, M. \& Schiffrin, J. D. Electrochemical charge injection into immobilized nanosized gold particle ensembles: potential modulated transmission and reflectance spectroscopy. Langmuir 15, 866-871 (1999).

21 Ung, T., Liz-Marzan, M. L. \& Mulvaney, P. Optical properties of thin films of Au@SiO2 particles. J. Phys. Chem. B 105, 3441-3452 (2001).

22 Hicks, F. J., Seok-Shon, Y. \& Murray, W. R. Layer-by-layer growth of polymer/nanoparticle films containing monolayer-protected gold clusters. Langmuir 18, 2288-2294 (2002).

23 Fu, Y., Xu, H., Bai, S., Quu, D., Sun, J., Wang, Z. \& Zhang, X. Fabrication of a stable polyelectrolyte/Au nanoparticles multilayer film. Makromol. Rapid. Commun. 23, 256-259 (2002).

24 Ferreyra, N., Coche-Guerente, L., Fatisson, J., Teijelo, L.M. \& Labbe, P. Layer-by-layer self-assembled multilayers of redox polyelectrolytes and gold nanoparticles. Chem. Commun. 2056-2057 (2003).

25 Liljeroth, P., Quinn, B. M., Ruiz, V. \& Kontturi, K. Charge injection and lateral conductivity in monolayers of metallic nnanoparticles. Chem. Commun. 1570-1571 (2003).

26 Jiang, C., Markutsya, S. \& Tsukruk, V. V. Collective and individual plasmon resonances in nanoparticle films obtained by spin-assisted layer-by-layer assembly. Langmuir 20, 882-890 (2004).

27 Qi, Z., Honma, I., Ichihara, M. \& Zhou, H. Layer-by-layer fabrication and characterization of gold-nanoparticle/myoglobin nanocomposite films. Adv. Funct. Mater. 6, 377-386 (2006).

28 Niwa, M., Morikawa, M-a. \& Higashi, N. Discrimination between N- and C-termini of polypeptides by a two-dimensional array of helical poly(L-glutamic acid) rods on gold surfaces. Langmuir 15, 5088-5092 (1999).

29 Miyazawa, T. \& Blout, E. R. The infrared spectra of polypeptides in various conformations: amide I and II bands. J. Am. Chem. Soc. 83, 712-719 (1961).

30 Kinoshita, T., Hayashi, S. \& Yokogawa, Y. Preparation of a structural color forming system by polypeptide-based LB films. J. Photochem. Photobio. A 145, 101-106 (2001). 